\title{
Can preoperative magnetic resonance imaging replace intraoperative frozen sectioning in the evaluation of myometrial invasion for early-stage endometrial carcinoma?
}

\author{
Volkan Karataşlı ${ }^{1}$, Illker Çakır ${ }^{1}$, Hilal Şahin ${ }^{2}$, Duygu Ayaz ${ }^{3}$, Muzaffer Sancı ${ }^{1}$ \\ ${ }^{1}$ Department of Gynecologic Oncology,University of Health Sciences Tepecik Education and Research Hospital, Izmir, Turkey \\ ${ }^{2}$ Department of Radiology,University of Health Sciences Tepecik Education and Research Hospital, Izmir, Turkey \\ ${ }^{3}$ Department of Pathology,University of Health Sciences Tepecik Education and Research Hospital, Izmir, Turkey
}

\begin{abstract}
Objectives: To examine the performance of preoperative magnetic resonance imaging (MRI) and intraoperative frozen sectioning in the assessment of myometrial invasion during the early stages of endometrial cancer.

Material and methods: This retrospective study employed data from patients with endometrial cancer who were operated on between January 2013 and November 2018. Patients who underwent preoperative MRI and were of FIGO 2009 stage I were included in the study. Radiological staging and intraoperative staging by frozen sectioning were carried out. The data were analyzed to assess agreement of the overall results concerning myometrial invasion.

Results: In total, 222 patients were enrolled. Their mean age was $58.3 \pm 8.5$ years. The accuracy of MRI for the detection of myometrial invasion was $88.7 \%$ and its sensitivity, specificity, positive predictive value (PPV), and negative predictive value (NPV) were $91.6 \%, 82.1 \%, 92.2 \%$, and $80.9 \%$, respectively, with a kappa coefficient of 0.734 (95\% confidence interval [Cl], 0.684-0.784; $\mathrm{p}<0.001)$. The accuracy of intraoperative frozen sectioning was $94.4 \%$, and its sensitivity, specificity, PPV, and NPV were $97.7 \%, 85.7 \%, 94.7 \%$, and $93.4 \%$, respectively, with a kappa coefficient of 0.856 (95\% Cl, 0.812-0.900; $p<0.001)$. No significant difference in accuracy was observed between MRI and frozen sectioning $(p=0.057)$. MRI and frozen sectioning were sensitive for the detection of myometrial invasion, according to receiver operating curve analyses (areas under the curve, 0.869 and 0.917 , respectively; $\mathrm{p}<0.001$ ).

Conclusions: The assessment of myometrial invasion by preoperative MRI and intraoperative frozen sectioning during the early stages of endometrial carcinoma was highly accurate.
\end{abstract}

Key words: endometrial cancer; myometrial invasion; magnetic resonance imaging; MRI; frozen sectioning

Ginekologia Polska 2019; 90, 3: 128-133

\section{INTRODUCTION}

Endometrial cancer is the most common gynecological malignancy in the United States and other developed countries [1]. As the majority of patients present with abnormal vaginal bleeding, particularly during the postmenopausal period, most cases are diagnosed in the early stages [2]. The prognosis for early-stage disease is generally excellent [3].

Surgical staging of endometrial cancer is necessary [2]. Disease staging provides insight about prognosis and adjuvant treatment [2]. The FIGO 2009 stage I subgroup is defined according to the depth of myometrial invasion (stage
IA, no invasion or invasion $<50 \%$ of the myometrial thickness; stage IB, invasion $\geq 50 \%$ of the myometrial thickness) [4]. In addition, myometrial invasion determines the risks of extrauterine disease and lymph node metastasis [5]. Lymph node metastasis occurs in $30 \%$ of cases of deep myometrial invasion, but only $5 \%$ of cases with superficial myometrial invasion [5]. Systematic lymphadenectomy is advised as part of surgical staging for high-risk patients [2]. In contrast, previous reports indicate that systematic lymphadenectomy does not improve disease-free or overall survival during the early stages of the disease [6, 7]. Lymphadenectomy may 
cause complications, such as lymphedema and lymphocysts; thus, it should be avoided in low-risk patients [2]. The risk of recurrence can be estimated by assessing the depth of myometrial invasion during preoperative radiological evaluation or by intraoperative frozen sectioning.

Preoperative radiological examination has been used to assess endometrial cancer, and magnetic resonance imaging (MRI) is the recommended diagnostic imaging method [8]. The updated guidelines of European Society of Urogenital Radiology recommend MRI as the imaging modality of choice for the evaluation of disease extent in patients with newly diagnosed endometrial cancer [9]. The European Society for Medical Oncology (ESMO), European Society for Radiotherapy \& Oncology (ESTRO), and European Society of Gynaecological Oncology (ESGO) recommend preoperative MRI for the evaluation of myometrial invasion in patients with stage 1 endometrial cancer [2]. Thus, MRI can be used to assess myometrial invasion during the early disease stage $[8,10,11]$. However, several studies have shown that the prediction of myometrial invasion using MRI alone can be difficult [12, 13].

Intraoperative examination modalities for endometrial tumors have been evaluated previously [14]. Tumor assessment by frozen sectioning has been found to have greater importance than gross examination [15]. However, the accuracy of frozen sectioning can be low [16]. Furthermore, some patients cannot be evaluated preoperatively by MRI, and low image quality may affect the ability to estimate disease extent preoperatively. In such situations, intraoperative frozen sectioning may play an important role. In addition, older patients and those with medical comorbidities, and younger patients who want to preserve their fertility, are not eligible for the operation [17]. Thus, preoperative evaluation is more important in these patients. Additionally, systematic lymphadenectomy and optimal staging cannot be performed in some patients because of the morbidity of lymphadenectomy and the extent of surgery $[2,18]$. Preoperative clinical staging gains importance in these patient groups.

Unnecessary lymphadenectomy with complications and morbidity can be prevented by the preoperative and intraoperative evaluation of myometrial invasion during the early stages of the disease [10]. Under-staging can be avoided in high-risk patients [10], and inoperable cases can be staged clinically [2].

\section{Objectives}

The aim of this study was to examine the performance of preoperative MRI and intraoperative frozen sectioning in the assessment of myometrial invasion before surgical staging in patients with early-stage endometrial cancer in a high-volume tertiary cancer center employing experienced radiologists and pathologists in a gynecological oncology department.

\section{MATERIAL AND METHODS}

This retrospective study employed data from $337 \mathrm{pa}-$ tients with endometrial cancer who were diagnosed and operated on between January 2013 and November 2018 in a high-volume cancer center in western Turkey. Patients with advanced stages ( $\geq$ II) of the disease and those with synchronous tumors were excluded. Patients who did not undergo pelvic MRI and were scanned with abdominal computed tomography or positron emission tomography/computed tomography, cases that were diagnosed incidentally after hysterectomy and patients who were not performed frozen sectioning were excluded. In total, 222 patients who were diagnosed preoperatively by biopsy, underwent MRI in our center, and were optimally staged by gynecological oncologists, and performed frozen sectioning in our department were included in the study. This study was approved by the local institutional ethic committee.

All biopsy samples were obtained preoperatively by dilatation and curettage or pipelle sampling. Pelvic MRI was carried out with a 1.5 Tesla system (Siemens Avanto, Siemens Aera, GE Optima 360; Erlangen, Germany) and a six-channel body coil. The imaging protocol involved sagittal, axial, coronal, and oblique axial T2-weighted images without fat saturation, as well as precontrast and postcontrast (gadoteric acid, $0.1 \mathrm{mmol} / \mathrm{kg}$ ) T1-weighted fat saturated images in the axial plane. One radiologist with 8 years of experience with pelvic MRI evaluated the images before surgery. The degree of myometrial invasion was interpreted as superficial or deep ( $\geq 50 \%$ of the myometrium). Radiological stages were assigned according to the imaging findings.

After a multidisciplinary tumor board examined the preoperative results, the patients underwent surgical staging by gynecological oncologists. Explorative laparotomy or laparoscopy was performed, followed by hysterectomy and bilateral salpingoophorectomy. The specimens were taken to the pathology department for frozen sectioning, according to the preoperative risk stratification of the endometrial cancer [2]. The lymph nodes were dissected according to the frozen sectioning results and preoperative risk group. Pathologists examined hysterectomy specimens using a longitudinal section of the endometrial cavity and uterine cervix, and a horizontal section from the uterine fundus, in each case. After placing the specimen over a film of optimal cutting temperature medium on a cryostat, the tumor was frozen, cut into 5 - $\mu \mathrm{m}$ slices using a microtome, and then prepared for staining with hematoxylin and eosin for microscopic evaluation. Tumor histology, grade, and diameter, and depth of myometrial invasion (superficial or deep), were examined. The same pathologists with experience in gynecological oncology examined the slides used for the final pathological report. In cases of diagnostic discrepancy, another pathologist examined the slides. The tumor board evaluated the 


\section{Table 1. Clinical characteristics of the patients}

\begin{tabular}{|l|l|}
\hline Variables $\mathrm{n}[\%]$ & $\mathrm{N}=222$ \\
\hline Age, mean $\pm \mathrm{SD}$ & $58.3 \pm 8.5$ \\
\hline Parity, median [range] & $3(0-10)$ \\
\hline $\begin{array}{l}\text { Menopausal Status } \\
\quad \text { Premenopausal }\end{array}$ & \\
$\quad$ Postmenopausal & $37(16.7 \%)$ \\
\hline BMl, mean $\pm \mathrm{SD}\left(\mathrm{kg} / \mathrm{m}^{2}\right)$ & $185(83.3 \%)$ \\
\hline 30 kg/m2 [n, \%] & $34.3 \pm 6.0$ \\
\hline $\mathrm{CA} 125$, median $($ range) & $169(76.1 \%)$ \\
\hline $35 \mathrm{U} / \mathrm{mL}[\mathrm{n}, \%]$ & $17(2-800)$ \\
\hline
\end{tabular}

$\mathrm{SD}$ - standard deviation; $\mathrm{BMI}$ - body mass index, $\mathrm{kg} / \mathrm{m} 2 ; \mathrm{CA} 125$ - cancer antigen $125, \mathrm{U} / \mathrm{mL}$

postoperative results and final stages according to the FIGO 2009 classification to determine the optimal treatment [13]. No or $<50 \%$ myometrial invasion was considered to reflect stage IA, and $\geq 50 \%$ invasion was classified as stage IB, according to the FIGO 2009 classification [4].

The accuracy, specificity, sensitivity, positive predictive value (PPV), and negative predictive value (NPV) were calculated for the results of preoperative MRI and intraoperative frozen sectioning according to the final pathology results. The Cohen kappa statistic was used to examine the agreement of the overall results concerning myometrial invasion. Receiver operating characteristic (ROC) curves were used to evaluate the accuracy of the tests. SPSS software (ver. 21; SPSS Inc., Chicago, IL, USA) was used for the statistical analyses, and $p$-values $<0.05$ were considered to be significant.

\section{RESULTS}

In total, 222 patients with stage 1 endometrial carcinoma were enrolled in this study. The clinical characteristics of the patients are shown in Table 1. The mean age of the patients was $58.3 \pm 8.5$ years. Most of the patients were postmenopausal $(83.3 \%)$ and obese (body mass index $>30 \mathrm{~kg} / \mathrm{m}^{2}$; 76.1\%). Twenty-seven (12.2\%) patients had high (> $35 \mathrm{U} / \mathrm{mL}$ ) preoperative levels of serum cancer antigen (CA) 125.

The surgical and histopathological results are summarized in Table 2. Approximately $64 \%$ of the patients were treated by laparotomy, and the majority (86.1\%) of them underwent pelvic \pm para-aortic lymphadenectomy. The most common tumor histological type was endometrioid, and $58.1 \%$ of the tumors were of grade 2 . In total, 172 of 222 (77.5\%) patients had tumor sizes $>2 \mathrm{~cm}$. Lymphovascular space involvement (LVSI) was detected in $20.7 \%$ of the pathological specimens. The median sizes of tumors without and with LVSI were 3.5 (0.2-9) $\mathrm{cm}$ and $4.5(2-8) \mathrm{cm}$, respectively $(p=0.001)$. According to the final pathological reports, 155 (69.8\%) patients had stage IA and 67 (30.2\%) had stage IB disease. The median dissected pelvic lymph node count was 12 (0-53).
Table 2. Surgical and histopathological characteristics of the patients

\begin{tabular}{|c|c|}
\hline & n [\%] \\
\hline $\begin{array}{l}\text { Surgery } \\
\text { TAH + BS } \pm \text { O } \\
\text { TLH/ Robotic Hysterectomy + BS } \pm \text { O } \\
\text { TAH + BSO + Pelvic LND } \\
\text { TLH/ Robotic Hysterectomy + BSO + Pelvic LND } \\
\text { TAH + BSO + Pelvic LND + Para-aortic LND } \\
\text { TLH + BSO + Pelvic LND + Para-aortic LND }\end{array}$ & $\begin{array}{l}17(7.7) \\
36(16.2) \\
41(18.5) \\
34(15.3) \\
85(38.3) \\
9(4.0)\end{array}$ \\
\hline $\begin{array}{l}\text { Tumor Histological Type } \\
\text { Endometrioid } \\
\text { Serous/Clear Cell } \\
\text { Mixed* } \\
\text { Others (Carcinosarcoma, Adenosarcoma, ESS) }\end{array}$ & $\begin{array}{l}193(86.9) \\
8(3.6) \\
14(6.3) \\
7(3.2)\end{array}$ \\
\hline $\begin{array}{l}\text { Tumor Grade } \\
1 \\
2 \\
3\end{array}$ & $\begin{array}{l}65(29.3) \\
129(58.1) \\
28(12.6)\end{array}$ \\
\hline $\begin{array}{l}\text { Tumor Size, } \mathrm{cm} \text {, median [range] } \\
\qquad 2 \mathrm{~cm} \\
>2 \mathrm{~cm}\end{array}$ & $\begin{array}{l}3,5(0.2-9) \\
50(22.5) \\
172(77.5)\end{array}$ \\
\hline $\begin{array}{l}\text { LVSI } \\
\quad \text { None } \\
\text { Present }\end{array}$ & $\begin{array}{c}176(79.3) \\
46(20.7)\end{array}$ \\
\hline $\begin{array}{l}\text { FIGO Stage } \\
\text { IA } \\
\text { IB }\end{array}$ & $\begin{array}{l}155(69.8) \\
67(30.2)\end{array}$ \\
\hline Pelvic LN Count, median & $15(2-53)$ \\
\hline Para-aortic LN Count, median, [range] & $9(1-41)$ \\
\hline
\end{tabular}

$\mathrm{TAH}+\mathrm{BS} \pm \mathrm{O}-$ total abdominal hysterectomy and bilateral salpingectomy with or without oophorectomy; $\mathrm{TLH}+\mathrm{BS} \pm \mathrm{O}$ - total laparoscopic

hysterectomy and bilateral salpingectomy with or without oophorectomy; $\mathrm{TAH}+\mathrm{BSO}+$ Pelvic LND — total abdominal hysterectomy and bilateral salpingoophorectomy plus pelvic lymphadenectomy; TLH + BSO + Pelvic LND - total laparoscopic hysterectomy and bilateral salpingoophorectomy plus pelvic lymphadenectomy; TAH + BSO + Pelvic LND + Para-aortic LND - total abdominal hysterectomy and bilateral salpingoophorectomy plus pelvic and para-aortic lymphadenectomy; TLH + BSO Pelvic LND + Para-aortic LND - total laparoscopic hysterectomy and bilateral salpingoophorectomy plus pelvic and para-aortic lymphadenectomy; LN — lymph node

*endometrioid + mucinous, endometrioid + serous, endometrioid + clear cell ESS - endometrial stromal sarcoma

LVSI - lymphovascular space invasion

The median time between MRI and operation was 21 (7-52) days. Tables 3 and 4 define the relationships of radiological staging and frozen sectioning to the final pathological results. Table 5 shows the results for combined consideration of MRI and frozen sectioning. The correlation of MRI

\begin{tabular}{|c|c|c|c|c|}
\hline & & \multicolumn{2}{|l|}{ Stage } & \multirow{2}{*}{ Total } \\
\hline & & IA & IB & \\
\hline \multirow{2}{*}{ MRI } & IA & $142(64.0)$ & $12(5.4)$ & 154 (69.4) \\
\hline & IB & $13(5.9)$ & $55(2.8)$ & $68(30.6)$ \\
\hline \multicolumn{2}{|c|}{ Total } & 155 (69.8) & $67(30.2)$ & $222(100)$ \\
\hline
\end{tabular}




\begin{tabular}{|l|l|l|l|l|}
\multicolumn{4}{|c|}{ Table 4. Frozen sectioning and the final pathological results } \\
\hline \multirow{2}{*}{} & & Stage & \multicolumn{1}{|c|}{ Total } \\
\cline { 2 - 5 } & IA & IB & \\
\hline $\begin{array}{l}\text { Frozen } \\
\text { section }\end{array}$ & IA & $126(70.8)$ & $7(3.9)$ & $133(74.7)$ \\
\hline Total & $3(1.7)$ & $42(23.6)$ & $45(25.3)$ \\
\hline a $n, \%$ & $129(72.5)$ & $49(27.5)$ & $178(100)$ \\
\hline
\end{tabular}

Table 5. Preoperative magnetic resonance imaging (MRI) and additional intraoperative frozen sectioning and final pathological results $^{\mathrm{a}}$

\begin{tabular}{l|l|l|l|l|}
\hline & & \multicolumn{2}{l|}{ Stage } & \multicolumn{1}{l}{ Total } \\
\cline { 2 - 5 } & IA & IB & \\
\hline $\begin{array}{l}\text { MRI + Frozen } \\
\text { section }\end{array}$ & IA & $127(71.4)$ & $2(1.1)$ & $129(72.4)$ \\
\hline Total & IB & $2(1.1)$ & $47(26.4)$ & $49(27.5)$ \\
\hline${ }^{\mathrm{a}} \mathrm{n}, \%$ & & $129(72.5)$ & $49(27.5)$ & $178(100)$ \\
\hline
\end{tabular}

and frozen sectioning with the final pathological findings is shown in Table 6. The accuracy of MRI for the detection of myometrial invasion was $88.7 \%$, and its sensitivity, specificity, PPV, and NPV were $91.6 \%, 82.1 \%, 92.2 \%$, and $80.9 \%$, respectively, with a kappa coefficient of 0.734 (95\% confidence interval $[\mathrm{Cl}], 0.684-0.784 ; \mathrm{p}<0.001)$. The accuracy of intraoperative frozen sectioning was $94.4 \%$, and its sensitivity, specificity, PPV, and NPV were $97.7 \%, 85.7 \%, 94.7 \%$, and $93.4 \%$, respectively, with a kappa coefficient of $0.856(95 \%$ $\mathrm{Cl}, 0.812-0.900 ; \mathrm{p}<0.001)$. The rates of overdiagnosis were $8.4 \%$ for MRI and $2.3 \%$ for frozen sectioning. The rates of underdiagnosis were $17.9 \%$ for MRI and $14.3 \%$ for frozen sectioning. Preoperative MRI yielded 5.4\% false-negative and $5.9 \%$ false-positive results for the prediction of deep myometrial invasion. The false-negative and false-positive ratios for frozen sectioning were $3.9 \%$ and $1.7 \%$, respectively. No significant difference in accuracy was observed between MRI and frozen sectioning, according to McNemar's test $(p=0.057)$. When MRI and frozen sectioning were considered together, the accuracy was $97.8 \%$ and the sensitivity, specificity, PPV, and NPV were $98.5 \%, 95.9 \%, 98.5 \%$, and $95.9 \%$, respectively, with a kappa coefficient of $0.944(95 \%$ $\mathrm{Cl}, 0.916-0.972 ; \mathrm{p}=0.028$ ).

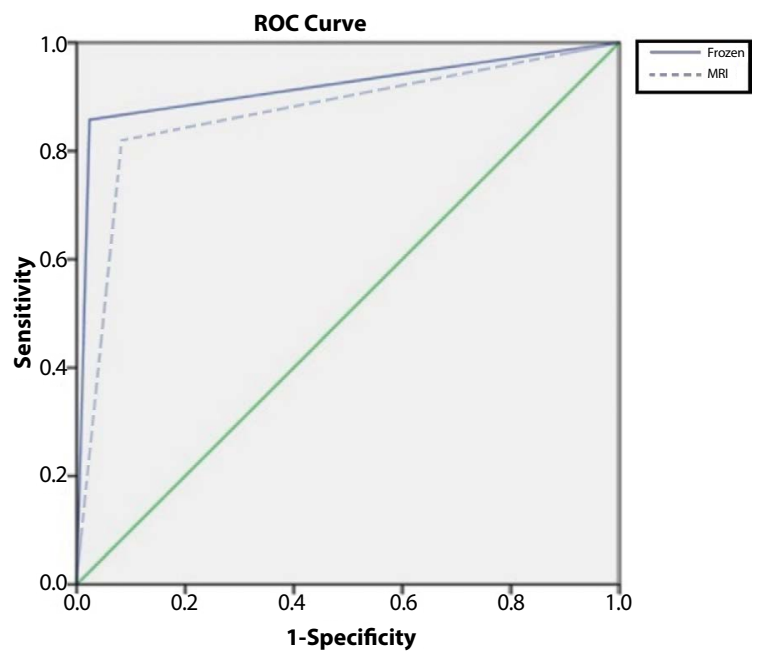

Figure 1. Receiver operating characteristic (ROC) curve for intraoperative frozen sectioning and magnetic resonance imaging (MRI) for the assessment of deep myometrial invasion (areas under the curve, 0.917 and 0.869 , respectively; $\mathrm{p}<0.001$ )

The ROC curves for the assessment of deep myometrial invasion are shown in Figure 1 for MRI and frozen sectioning. MRI and the frozen sectioning significantly detected myometrial invasion, according to the ROC curve analyses (areas under the curve, 0.869 and 0.917 , respectively; $p<0.001$ ).

\section{DISCUSSION}

Endometrial cancer is commonly diagnosed in postmenopausal women; the average age of patients in the United States is 63 years [3]. Most cases in our study group were postmenopausal and $>50$ years of age, consistent with the literature. Obesity is an important risk factor for endometrial carcinoma [19]. The majority of the patients in our study group were obese, and the morbid obesity rate was high. Preoperative elevated serum CA 125 levels (> $35 \mathrm{U} / \mathrm{mL}$ ) are associated with extrauterine disease [20]. Although the patients had early-stage disease, CA 125 levels were above the limit in $12.2 \%$ of cases in this study. Elevated CA 125 levels are correlated with increasing depth of myometrial invasion [20]. The median CA 125 level was higher in the deep myometrial invasion group, consistent with previous studies $[20,21]$.

\begin{tabular}{|c|c|c|c|c|c|c|c|c|}
\hline & Accuracy & $\begin{array}{l}\text { Kappa } \\
\text { Coefficient }\end{array}$ & Sensitivity & Specificity & PPV & NPV & $\begin{array}{l}\text { Over- } \\
\text { Diagnosis }\end{array}$ & $\begin{array}{l}\text { Under- } \\
\text { Diagnosis }\end{array}$ \\
\hline MRI & $88.7 \%$ & 0.734 & $91.6 \%$ & $82.1 \%$ & $92.2 \%$ & $80.9 \%$ & $8.4 \%$ & $17.9 \%$ \\
\hline Frozen Section & $94.4 \%$ & 0.856 & $97.7 \%$ & $85.7 \%$ & $94.7 \%$ & $93.4 \%$ & $2.3 \%$ & $14.3 \%$ \\
\hline $\begin{array}{l}\text { MRI + Frozen } \\
\text { Section }\end{array}$ & $97.8 \%$ & 0.944 & $98.5 \%$ & $95.9 \%$ & $98.5 \%$ & $95.9 \%$ & $1.6 \%$ & $4.1 \%$ \\
\hline
\end{tabular}

PPV — positive predictive value; NPV— negative predictive value 
Surgery for endometrial cancer can be performed with laparotomy or laparoscopy [22]. As minimally invasive surgery is recommended because of its lesser morbidity, the laparoscopic surgery rate is increasing with increases in minimally invasive surgical skills and facilities [22]. Only patients at high risk of recurrence should undergo lymphadenectomy [2]. This procedure can cause lower extremity lymphedema, lymphocysts, and surgical morbidities [4]. In this study, the risk classification was estimated using age, tumor histology, grade, and radiological and frozen sectioning findings. Although the patients in this study group had early-stage disease, pelvic or para-aortic lymphadenectomy was applied in the majority of cases. This pattern can be explained by the change in our approach since the January 2016 ESMO/ESTRO/ESGO consensus conference report, with no lymphadenectomy performed in a low-risk patient [2]. The majority of patients with endometrial cancer are diagnosed at the early stage [3]. All patients in this study were diagnosed at an early stage, and most cases were diagnosed as stage IA.

MRI is an appropriate imaging modality for the detection of myometrial invasion, extrauterine disease, and lymph node metastasis in patients with endometrial cancer [8]. Clinical staging can be estimated according to the radiological evaluation. Lin et al. [23] reported high sensitivity and specificity levels for MRI and an accuracy of $94 \%$. However, other authors reported that the accuracy of MRI can be as low as $65 \%$ [24]. The sensitivity and accuracy levels obtained in this study were higher than in previous reports. The kappa coefficients also showed good correlations; thus, radiologists' experience is important for the interpretation of imaging results.

The intraoperative pathological examination of frozen-sectioned specimens is convenient for the detection of myometrial invasion [14, 15, 25]. However, Case et al. [16] reported that the accuracy of frozen sectioning is low (67\%), posing a risk for under-staging, which can lead to suboptimal treatment, so they advised surgical staging for all patients with endometrial cancer. In our study, the accuracy of frozen sectioning was high, which is thought to be related to the skill level of the pathologists at our center, who have more than 10 years of experience in gyneco-pathology. In addition, a high correlation was observed between frozen sectioning findings and the final pathological results for deep myometrial invasion.

Tanaka et al. [26] and Kisu et al. [27] reported that frozen sectioning has a higher correlation rate than does MRI. They mentioned that diffusion-weighted MRI can have the same diagnostic precision as frozen sectioning [11]. In our study, the accuracies of MRI and frozen sectioning were similar. Additional frozen sectionings are recommended when MRI is positive or negative for the presence of myometrial invasion [27].
In our study, the correlation ratios were high when MRI and additional frozen sectioning results were interpreted together. In addition, over-diagnosis and under-diagnosis rates were lower with this approach.

Some patients cannot be operated on due to advanced age, morbid obesity, and/or medical comorbidities [2, 17]. In addition, some young patients wish to preserve their fertility $[2,28]$. Thus, surgical staging cannot be performed and intra-abdominal or extrauterine spread of the disease cannot be seen in these cases. Clinical evaluation and preoperative MRI may be more important for these non-surgical patient groups.

A limitation of this study is its retrospective design. However, the high accuracy and correlation rates from a high-volume center, as well as the involvement of experienced radiologists and pathologists, support the importance of radiological and intraoperative evaluation. As diffusion-weighted MRI can increase the correlation rate, diffusion-weighted images have also been used recently.

\section{CONCLUSIONS}

The detection of myometrial invasion before surgery and the final pathology results reveal the need for lymphadenectomy to predict the prognosis, and are also thought to be very important for non-surgical patients. The assessment of myometrial invasion by preoperative MRI and intraoperative frozen sectioning during the early stages of endometrial carcinoma was highly accurate. Preoperative MRI can have the same diagnostic precision as frozen sectioning. The assessment of myometrial invasion by preoperative MRI and additional intraoperative frozen sectionings provided the most accurate results. So over-diagnosis and under-diagnosis rates were lower with this approach.

\section{Acknowledgements}

None.

\section{REFERENCES:}

1. Miller KD, Siegel RL, Lin CC, et al. Cancer statistics, 2016. CA Cancer J Clin. 2016; 66(1): 7-30, doi: 10.3322/caac.21332, indexed in Pubmed: 26742998.

2. Colombo N, Creutzberg C, Amant F, et al. ESMO-ESGO-ESTRO Endometrial Consensus Conference Working Group. ESMO-ESGO-ESTRO Consensus Conference on Endometrial Cancer: diagnosis, treatment and follow-up. Ann Oncol. 2016; 27(1): 16-41, doi: 10.1093/annonc/mdv484, indexed in Pubmed: 26634381.

3. SEER Cancer Stat Facts: Uterine Cancer.National Cancer Institute. Bethesda M. https://www.seer.cancer.gov/statfacts/html/corp. html (13.12.2018).

4. Pecorelli S. Revised FIGO staging for carcinoma of the vulva, cervix, and endometrium. Int J Gynaecol Obstet. 2009; 105(2): 103-104, indexed in Pubmed: 19367689.

5. Larson DM, Connor GP, Broste SK, et al. Prognostic significance of gross myometrial invasion with endometrial cancer. Obstet Gynecol. 1996; 88(3): 394-398, doi: 10.1016/0029-7844(96)00161-5, indexed in Pubmed: 8752246.

6. Kitchener H, Swart AMC, Qian Q, et al. ASTEC study group. Efficacy of systematic pelvic lymphadenectomy in endometrial cancer (MRC 
ASTEC trial): a randomised study. Lancet. 2009; 373(9658): 125-136, doi: 10.1016/S0140-6736(08)61766-3, indexed in Pubmed: 19070889.

7. Turkler C, Kulhan M, Kulhan NG, et al. Role of lymphadenectomy in disease-free and overall survival on low risk endometrium cancer patients. Ginekol Pol. 2018; 89(6): 311-315, doi: 10.5603/GP.a2018.0053, indexed in Pubmed: 30010179.

8. Kinkel K, Kaji Y, Yu KK, et al. Radiologic staging in patients with endometrial cancer: a meta-analysis. Radiology. 1999; 212(3): 711-718, doi: 10.1148/radiology.212.3.r99au29711, indexed in Pubmed: 10478237.

9. Nougaret S, Horta M, Sala E, et al. Endometrial Cancer MRI staging: Updated Guidelines of the European Society of Urogenital Radiology. Eur Radiol. 2019; 29(2): 792-805, doi: 10.1007/s00330-018-5515-y, indexed in Pubmed: 29995239.

10. Sanjuán A, Cobo T, Pahisa J, et al. Preoperative and intraoperative assessment of myometrial invasion and histologic grade in endometrial cancer: role of magnetic resonance imaging and frozen section. Int J Gynecol Cancer. 2006; 16(1): 385-390, doi: 10.1111/j.1525-1438.2006 .00414.x, indexed in Pubmed: 16445663.

11. Gallego JC, Porta A, Pardo MC, et al. Evaluation of myometrial invasion in endometrial cancer: comparison of diffusion-weighted magnetic resonance and intraoperative frozen sections. Abdom Imaging. 2014; 39(5): 1021-1026, doi: 10.1007/s00261-014-0134-9, indexed in Pubmed: 24699937.

12. Chung HH, Kang SB, Cho JY, et al. Accuracy of MR imaging for the prediction of myometrial invasion of endometrial carcinoma. Gynecol Oncol. 2007; 104(3): 654-659, doi: 10.1016/j.ygyno.2006.10.007, indexed in Pubmed: 17095081

13. Scoutt LM, McCarthy SM, Flynn SD, et al. Clinical stage I endometrial carcinoma: pitfalls in preoperative assessment with MR imaging. Work in progress. Radiology. 1995; 194(2): 567-572, doi: 10.1148/radiology.194.2.7824739, indexed in Pubmed: 7824739.

14. Stephan JM, Hansen J, Samuelson M, et al. Intra-operative frozen section results reliably predict final pathology in endometrial cancer. Gynecol Oncol. 2014; 133(3): 499-505, doi: 10.1016/j.ygyno.2014.03.569, indexed in Pubmed: 24699308.

15. Alcazar JL, Dominguez-Piriz J, Juez L, et al. Intraoperative Gross Examination and Intraoperative Frozen Section in Patients With Endometrial Cancer for Detecting Deep Myometrial Invasion: A Systematic Review and Meta-analysis. Int J Gynecol Cancer. 2016; 26(2): 407-415, doi: 10.1097/IGC.0000000000000618, indexed in Pubmed: 26807569.

16. Case AS, Rocconi RP, Straughn JM, et al. A prospective blinded evaluation of the accuracy of frozen section for the surgical management of endometrial cancer. Obstet Gynecol. 2006; 108(6): 1375-1379, doi: 10.1097/01. AOG.0000245444.14015.00, indexed in Pubmed: 17138769.
17. Pal N, Broaddus RR, Urbauer DL, et al. Treatment of Low-Risk Endometrial Cancer and Complex Atypical Hyperplasia With the Levonorgestrel-Releasing Intrauterine Device. Obstet Gynecol. 2018; 131(1): 109-116, doi: 10.1097/AOG.0000000000002390, indexed in Pubmed: 29215513.

18. Scribner DR, Walker JL, Johnson GA, et al. Surgical management of early-stage endometrial cancer in the elderly: is laparoscopy feasible? Gynecol Oncol. 2001; 83(3): 563-568, doi: 10.1006/gyno.2001.6463, indexed in Pubmed: 11733973.

19. Kaaks R, Lukanova A, Kurzer MS. Obesity, endogenous hormones, and endometrial cancer risk: a synthetic review. Cancer Epidemiol Biomarkers Prev. 2002; 11(12): 1531-1543, indexed in Pubmed: 12496040.

20. Hsieh $\mathrm{CH}$, ChangChien CC, Lin H, et al. Can a preoperative CA 125 level be a criterion for full pelvic lymphadenectomy in surgical staging of endometrial cancer? Gynecol Oncol. 2002; 86(1): 28-33, indexed in Pubmed: 12079296.

21. Ünsal M, Kimyon Comert G, Karalok A, et al. The preoperative serum CA125 can predict the lymph node metastasis in endometrioid-type endometrial cancer. Ginekol Pol. 2018; 89(11): 599-606, doi: 10.5603/GP.a2018.0103, indexed in Pubmed: 30508211.

22. Wright J, BurkeW, Tergas A, et al. Comparative Effectiveness of Minimally Invasive Hysterectomy for Endometrial Cancer. Journal of Clinical Oncology. 2016; 34(10): 1087-1096, doi: 10.1200/jco.2015.65.3212.

23. Lin $\mathrm{G}, \mathrm{Ng} \mathrm{KK}$, Chang $\mathrm{CJ}$, et al. Myometrial invasion in endometrial cancer: diagnostic accuracy of diffusion-weighted 3.0-T MR imaging-initial experience. Radiology. 2009; 250(3): 784-792, doi: 10.1148/radiol.2503080874, indexed in Pubmed: 19244045.

24. Haldorsen IS, Husby JA, Werner HMJ, et al. Standard 1.5-T MRI of endometrial carcinomas: modest agreement between radiologists. Eur Radiol. 2012; 22(7): 1601-1611, doi: 10.1007/s00330-012-2400-y, indexed in Pubmed: 22453859.

25. Quinlivan JA, Petersen RW, Nicklin JL. Accuracy of frozen section for the operative management of endometrial cancer. BJOG. 2001; 108(8): 798-803, indexed in Pubmed: 11510702.

26. Tanaka T, Terai Y, Ono YJ, et al. Preoperative MRI and intraoperative frozen section diagnosis of myometrial invasion in patients with endometrial cancer. Int J Gynecol Cancer. 2015; 25(5): 879-883, doi: 10.1097/IGC.0000000000000470, indexed in Pubmed: 25950131.

27. Kisu I, Banno K, Lin LY, et al. Preoperative and intraoperative assessment of myometrial invasion in endometrial cancer: comparison of magnetic resonance imaging and frozen sections. Acta Obstet Gynecol Scand. 2013; 92(5): 525-535, doi: 10.1111/aogs.12048, indexed in Pubmed: 23163480.

28. Carneiro MM, Lamaita RM, Ferreira MC, et al. Fertility-preservation in endometrial cancer: is it safe? Review of the literature. JBRA Assist Reprod. 2016; 20(4): 232-239, doi: 10.5935/1518-0557.20160045, indexed in Pubmed: 28050959. 